\title{
Metrology for LNG custody transfer and transport fuel applications
}

Gerard Nieuwenkamp ${ }^{1, *}$, Nikola Pelevic ${ }^{1}$, Jianrong Li $^{1}$, Oliver Büker ${ }^{2}$, Karine Arrhenius ${ }^{2}$, Krister Stolt $^{2}$, Kurt Rasmussen ${ }^{3}$, Johan Kondrup ${ }^{3}$, Rémy Maury ${ }^{4}$, Markus Richter ${ }^{5}$, Rafael Lentner ${ }^{5}$, P. Alberto Albo ${ }^{6}$, Simona Lago ${ }^{6}$, Andrew Brown ${ }^{7}$, Arul Murugan $^{7}$, Björn Gieseking ${ }^{7}$, Jordan Tompkins ${ }^{7}$, Peter Eilts ${ }^{8}$, Lennart Klare ${ }^{8}$, Kai Moshammer ${ }^{9}$, Arnas Lucassen ${ }^{9}$ and Kianoosh Hadidi $^{10}$

${ }^{1}$ VSL, Thijsseweg 11, 2629 JA Delft, the Netherlands

${ }^{2}$ RISE Research Institutes of Sweden, Brinellgatan 4, 50462 Boräs, Sweden

${ }^{3}$ FORCE technology, Park Allé 345, 2605 Brøndby, Denmark

${ }^{4}$ CESAME exadébit, 43 Route de l'aerodrome, 86036 Poitiers, France

${ }^{5}$ RUB Ruht-Universität Bochum, 44780 Bochum, Germany

${ }^{6}$ INRiM, Strada delle Cacce 91, 10135 Torino, Italy

${ }^{7}$ NPL, Hampton Road, Teddington Middlesex TW11 0LW, United Kingdom

${ }^{8}$ TU Braunschweig, Hermann-Blenk Straße 42, 38108 Braunschweig, Germany

${ }^{9}$ PTB, Bundesallee 100, 38116 Braunschweig, Germany

${ }^{10}$ Justervesenet, Fetveien 99, 2007 Kjeller, Norway

\begin{abstract}
Liquefied natural gas (LNG) is a strategic and, in case of long distances, more economical alternative for pipeline natural gas. LNG is seen as the only alternative for diesel fuel for heavy duty transport (trucks, ships), with a better $\mathrm{CO}_{2}$ footprint and less noise produced by the engine. To enable the large scale roll-out of LNG as a transport fuel, a well established metrological infrastructure for LNG has to be realized. Within the framework of the EMRP Joint research Project ENG60 "Metrology for LNG" (LNG II), a number of metrological challenges are faced. The goals are to provide traceable mass and volume flow calibrations up to $400 \mathrm{~m}^{3} \mathrm{LNG} / \mathrm{h}$, to improve and validate sampling techniques for composition measurements, to improve the measurement of the LNG density and to prepare and validate an algorithm for the determination of the methane number. This paper gives an overview of the objectives that have been achieved and it provides an outlook to future activities that will be performed within a.o. the EMPIR 16ENG09 LNG III project.
\end{abstract}

\section{Introduction}

In view of a worldwide rise in energy consumption and the resulting increasing emission of greenhouse gases, there is a growing demand for natural gas. Liquefied natural gas (LNG) is a strategic and, in case of long distances, more economical alternative for pipeline natural gas. LNG is also addressed in the "Clean Power for Transport package" as one of the pillars of the European Clean fuel strategy [1]. LNG is seen as the only alternative for diesel for heavy duty transport (ships, trucks), having a better $\mathrm{CO}_{2}$ footprint and less noise produced by the engine.

In recent years, the trade of pipeline natural gas declined, while LNG shipment trade increased [2,3]. The transportation of LNG by ship is, in comparison to pipeline transportation, nearly independent from political conditions and is expected to continue to grow in volume and to become as important (or even more) as pipeline transport of natural gas.
However, LNG as a product is technically and metrologically much more challenging then "common" natural gas. LNG is a liquefied mixture of hydrocarbons and it is stored and used at a temperature of approx. -162 ${ }^{\circ} \mathrm{C}$, close to its boiling point. During transport the composition, and thereby the quality and all related physical and chemical properties of the LNG, will change due to evaporation of the lighter components, such as methane, from the LNG.

To enable the broad scale use of $\mathrm{LNG}$, reliable measurements are needed in the complete custody transfer chain from source to engine. A validated and well established metrological infrastructure for LNG related measurements has to be in place, to support fair trade and scientific developments.

\section{Energy measurement (custody transfer)}

The trade of LNG is based on energy measurement. This means, when LNG is loaded or uploaded from a ship, the

Corresponding author: gnieuwenkamp@vsl.nl

C) The Authors, published by EDP Sciences. This is an open access article distributed under the terms of the Creative Commons Attribution License 4.0 (http://creativecommons.org/licenses/by/4.0/). 
energy content is the crucial quantity. This energy content is commonly calculated from the volume, density and gross calorific value, according to the following equation from the GIIGNL handbook [4]:

$$
E=V_{L N G} \cdot D_{L N G} \cdot G C V_{L N G}-E_{\text {gas displaced }}
$$

Where:

$$
\begin{array}{ll}
E & \text { Energy transferred (MMBTU) } \\
V_{L N G} & \text { Volume of the LNG }\left(\mathrm{m}^{3}\right) \\
D_{L N G} & \text { Density of the LNG }\left(\mathrm{kg} / \mathrm{m}^{3}\right) \\
G C V_{L N G} & \text { Gross calorific value }(\mathrm{MMBTU} / \mathrm{kg}) \\
E_{\text {gas displaced }} & \text { Energy of the gas displaced during transfer } \\
& \text { (MMBTU) }
\end{array}
$$

Table 1 shows some typical estimated measurement uncertainties for the quantities in equation 1.

Table 1 : Estimated measurement uncertainties according to the GIIGNL handbook

\begin{tabular}{cc}
\hline Source of uncertainty & $\begin{array}{c}\text { Typical uncertainty values } \\
\boldsymbol{U}(\boldsymbol{E}) / \boldsymbol{E}(\boldsymbol{k}=2)\end{array}$ \\
\hline Volume & $0.20 \%-0.54 \%$ \\
Density & $0.46 \%$ \\
GCV & $0.04 \%-0.07 \%$ \\
E $_{\text {gas,displaced }}$ & $0.003 \%$ \\
Energy (total): & $0.50 \%-0.74 \%$ \\
\hline
\end{tabular}

As can be seen in the table, the overall measurement uncertainty in determining the transferred LNG energy content is in a best case scenario in the order of $0.5 \%$ relative (based on $\mathrm{k}=2$ ). In combination with the high quantities of LNG and the associated high value, this uncertainty represents a potential economic risk for the buyer and/or the seller of the product of many M€ per year. Reduction of the measurement uncertainty would be highly beneficial to the LNG market.

The (static) volume uncertainty of an LNG tank varies typically in the range of $0.20-0.54 \%(\mathrm{k}=2)$ see table 1 . There is almost a universal agreement that dynamic LNG flow metering has the potential to provide higher accuracy. In case LNG flow meters could be calibrated under operational conditions, measurement uncertainties in the range of $0.15-0.20 \%(\mathrm{k}=2)$ should be possible.

Also, a more accurate determination of the density offers a great potential for a reduction of the measurement uncertainty. An improved Equation of State (EoS) would enable a better prediction of the LNG density in case the measurement instruments are calibrated with another species and/or at another temperature.

Another way to obtain the density is by calculation from its composition. The composition of (L)NG can be analyzed by techniques such as gas chromatography and Raman spectroscopy, however, reference materials to calibrate the instruments and to validate the sampling techniques used for the analyses are lacking. From the composition also the GCV can be calculated.

The uncertainty resulting from the calculation of the energy of the gas displaced is negligible and will not be taken into account in this project.

\section{LNG calibration facility}

Conventional volumetric gauging calibration techniques are the state of the art measurement principle for the volumetric determination of LNG ship cargo transfer. These conventional methods have several disadvantages. The level measurement only takes place before and after the loading or unloading process. For a proper correction regarding operation conditions so-called tank gauge tables are used. Tank gauge tables contain of correction tables for list and trim (inclinometer), volume corrections for the tank inclusive tank deformation, process temperatures and other corrections. The alternative use of flow meters appears to be very attractive to industry. In the meanwhile flow meters are used even more as secondary standards at some LNG terminals. These flow meters are commercially available. However, they are hardly used for custody transfer due to lack of direct traceability to a LNG flow standard at this scale and lack of an ISO standard. The need for such development is particularly high for small- and midscale distributions and the application of LNG as a transport fuel where there is no alternative to flow metering.

For this reason the development of novel and traceable calibration standards for in-line LNG mass and volume flow is an important part to support the use of flow metering technology throughout the LNG production and distribution chain. An essential component of the new mid-scale primary LNG flow calibration standard (see Figure 1 and 2) is the low-scale LNG flow calibration standard for flow rates up to $25 \mathrm{~m}^{3} / \mathrm{h}(11250 \mathrm{~kg} / \mathrm{h})$ and measurement uncertainties $(\mathrm{k}=2)$ of $0.10-0.13 \%$, developed and validated within the previous EMRP joint research project (JRP) ENG03, in the period from $2009-2013$.

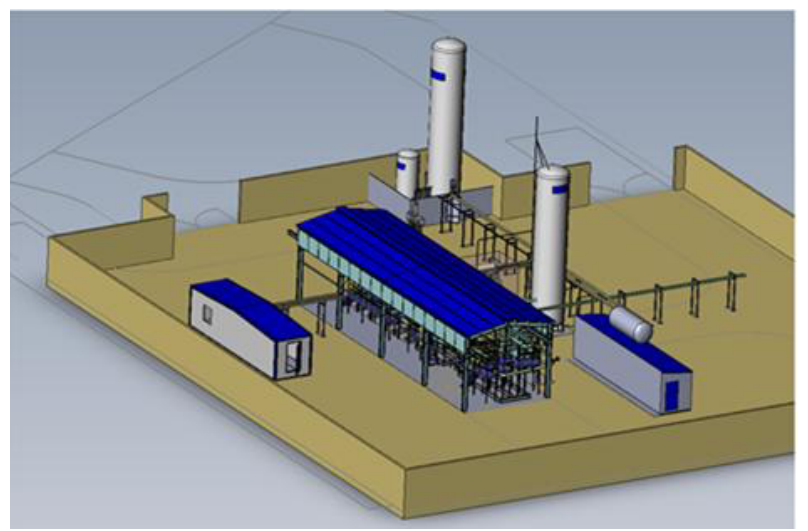

Fig. 1. Schematic Overview of the new mid-scale LNG flow facility (the building on the bottom right is the lowscale primary standard, the larger building in the middle contains the meter run package as well as the reference meters and working standards, the building on the left side of the figure is the control room). 


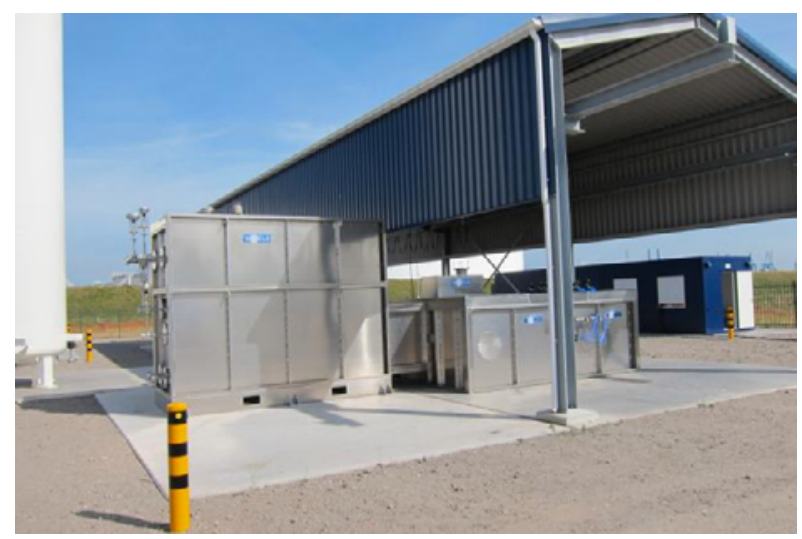

Fig. 2. Picture of the current state of the new mid-scale LNG flow facility at the Maasvlakte, in the Rotterdam harbour area in the Netherlands.

The new mid-scale primary standard will build the next step towards full traceability of large scale flow metering. This concerns the design, construction and validation of a mid-scale mass and volume flow calibration standard operating for flow rates up to 200 $\mathrm{m}^{3} / \mathrm{h}$, with a possible extension to $400 \mathrm{~m}^{3} / \mathrm{h}$. Targeting on a measurement uncertainty of $0.15 \%$ for mass and $0.20 \%$ for volume. A (mass) flow meter operating with a measurement uncertainty $(k=2)$ of e.g. $0.30 \%$ (incl. potential installation effects) will be a clear improvement compared to volume determined with tank gauging and conversion to mass through density, with an estimated combined measurement uncertainty of around $0.50-$ $0.70 \%(k=2)$. With use of Coriolis mass flow meters (CFM) and Ultrasonic flow meters (UFM) two types of flow meters with great potential for use in custody transfer will be investigated. Both types of flow meters have their advantages and disadvantages. CMFs measure directly mass flow and do not require a density calculation. This is especially useful since the gross calorific value is expressed per unit mass. However, CMFs have size and pressure drop limitations. UFMs in turn require a density calculation but have no size limitations and providing only a very low pressure drop. UFMs are therefore well suited for transfer measurements of large volume of LNG. In the course of the project, the preparation of a new ISO standard for flow metering systems has started in ISO/TC 28 WG 20. This new standard will accelerate the application of flow meters in LNG custody transfer and lead to smaller uncertainties and simpler operations. Drafted standard will be ready for voting by the end of 2017 . Finally the new mid-scale LNG facility will be used to carry out R\&D programs to validate models for extrapolating flow meter calibrations from low to high flow rates and from water to LNG. In addition, currently LNG flow metering techniques are relying on water calibrations. The facility will also be used for research results on flow meter installation effects.

In addition, a prototype of Laser Doppler Velocimeter (LDV) developed in the (JRP) ENG03 project will be validated under cryogenic industrial conditions with a relative uncertainty of $0.2 \%$. This technique forms an alternative route for establishing traceability of LNG volume flow measurements and strengthens the metrological framework.

\section{Density}

Besides volume determination, reliable LNG density predictions with small uncertainties are a prerequisite for the custody transfer of energy. Therefore, the aim is to measure LNG densities at the Ruhr-University Bochum (RUB) and at the national metrology institute of Italy (INRiM) in order to provide an improved model for LNG density prediction.

\subsection{LNG density measurements using single- sinker densimeter based on the Archimedes (buoyancy) principle}

An LNG densimeter was developed at RUB, based on the well-known single-sinker density measurement principle in conjunction with a magnetic suspension coupling. This principle was combined with a special VLE-cell allowing for liquid-density measurements at cryogenic temperatures without changing the composition of the liquid phase. In combination with a new way of operating the apparatus (e.g., supercritical filling), this feature enables measurements, which are much less affected by measurement uncertainties of the liquid-phase composition than measurements with other previous systems. After minimization of a well-known distorting effect - the force-transmission error of the magnetic suspension coupling - density measurements in the homogeneous liquid region of cryogenic liquid mixtures were carried out with relative combined expanded uncertainties of $0.044 \% \quad(k=2)$ [5]. The densimeter has been deliberately revised to reduce the uncertainty to a value of $0.030 \%$. The detailed design of the core apparatus of the new densimeter is shown by a sectional drawing in Fig. 3.

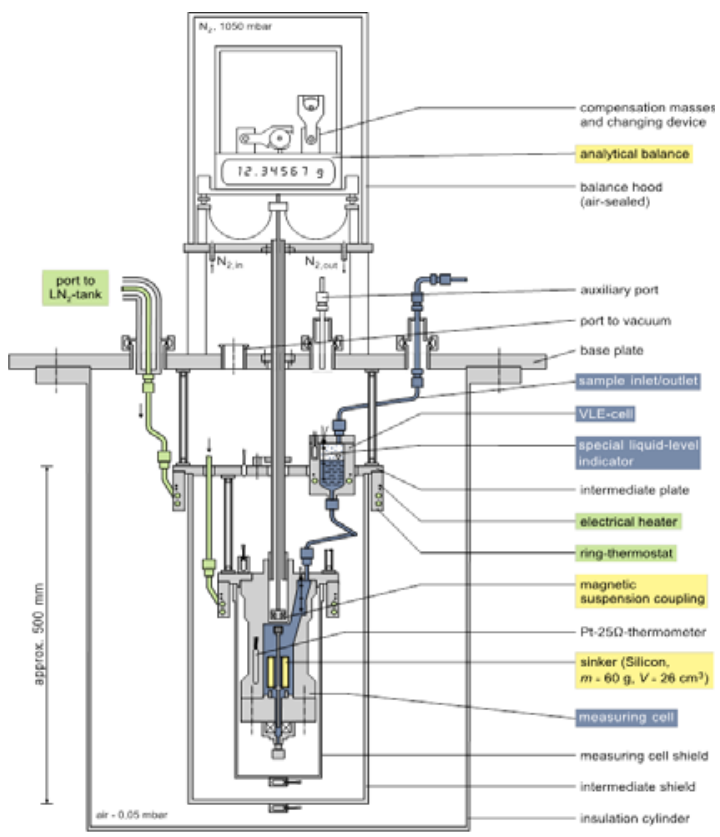

Fig. 3. Schematic diagram of the core apparatus of the densimeter for cryogenic liquid mixtures. 
Densities of six different synthetic LNG mixtures were measured over a temperature range from 105 to $135 \mathrm{~K}$ at pressures up to $9 \mathrm{MPa}$. The measurements include saturated liquid densities and homogeneous liquid states as well as supercritical states. The maximum relative deviations of the measured LNG densities from values calculated with the GERG-2008 EOS [6] are between $+0.05 \%$ and $+0.22 \%$, and the experimental saturated liquid densities agree well with the GERG-2008 EOS, in most cases even better with the "Revised Klosek and McKinley Method" (RKM).

\subsection{Improvement of a selected equation of state (EOS) for the calculation of LNG densities}

The LNG density is crucial for the custody transfer, and in most cases the density is calculated according to the GIIGNL handbook [4]. Therein, the RKM is recommended for density calculation, which is a pressureindependent equation to calculate saturated liquid densities within the temperature range relevant for the LNG custody transfer $(T<115 \mathrm{~K})$. Today, it is known that this particular equation is in need of improvement with regard to pressure-dependent density calculations and the corresponding uncertainties. By comparing the new experimental data sets with the relevant equations of state (e.g., RKM and GERG-2008 EOS), RUB developed an enhanced Revised Klosek-McKinleymethod (ERKM). The original RKM was supplemented by a new pressure-dependent term. The uncertainties of density calculations with the new ERKM are (independent of pressure) $0.10 \%$ for $100 \mathrm{~K} \leq T \leq 115 \mathrm{~K}$ and $0.15 \%$ for $115 \mathrm{~K}<T \leq 135 \mathrm{~K}$. RUB implemented the ERKM in their software tool called 'TREND', which also includes other models for the calculation of the thermodynamic properties of LNG.

\subsection{LNG ultrasonic speed-of-sound and density sensor}

INRiM developed a new type of sensor, which simultaneously determines densities and speed-of-sound (SoS) of LNG samples in the liquid phase at different cryogenic temperatures $(91<(\mathrm{T} / \mathrm{K})<110)$. The physical measurement principle consists of a dynamic measurement completely different and independent from densimeter developed by RUB, so that the intercomparison and mutual validation of the experimental results is feasible.

The new SoS sensor (see Fig. 4) with a relative measurement uncertainty $(k=2)$ of approx. $0.05 \%$ works on the principle of the double pulse-echo technique at cryogenic temperature and with a closed-loop cryostat appropriate to thermostat the sensor. This design differs from commercial ultrasonic flow-meters because the cell works completely immersed into the liquid (LNG), in this way density of the LNG sample is obtained (relative measurement uncertainty $(k=2)$ approx. $0.2 \%)$ by measuring its acoustic impedance $Z$ and the sound velocity $w, \rho=Z / w$.

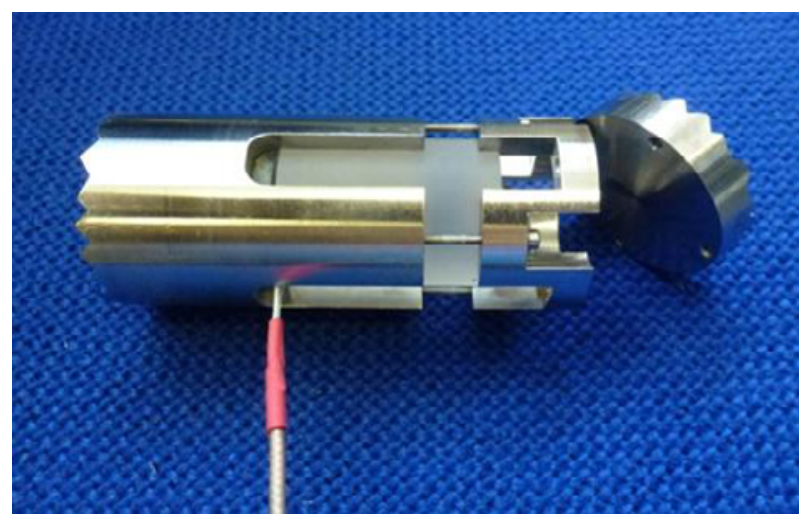

Fig. 4. INRiM's first prototype sensor for the simultaneous measurement of the density and speed of sound of a fluid based on the determination of the acoustic impedance of the sample.

Preliminary results obtained in n-nonane, n-undecane, toluene and water [7] are within $0.2 \%$ and thus show a good agreement between the measured density and literature data. Then the sensor will be validated at cryogenic temperature (in the LNG range) inside of the new laboratory cryostat. With the developed SoS sensor, densities and speed-of-sound of methane and, additionally, of LNG-like mixtures will be determined in liquid phase.

\section{Sampling and composition measurement}

The accurate and traceable determination of the LNG composition is crucial for the custody transfer as it allows for the accurate calculation of the physical properties as well as the methane number - both used in transport and trade. For natural gas the most accurate method for measuring the composition is gas chromatography (GC), however in the case of LNG the sampling from the liquid into a homogenous gaseous phase for analysis is challenging. An alternative envisaged for on-line testing in the LNG pipeline stream is Raman spectroscopy. Combined measurements using both GC and Raman will be performed when the calibration facility in the Netherlands is operational.

\subsection{Mobile and compact sampler}

One aim is to address the aforementioned issues by investigating methods to sample and vaporise LNG in a representative, homogeneous and reproducible fashion. In order to achieve this, a mobile and compact sampler has been developed and has been tested and validated on-site at an LBG storage facility in Gothenburg, Sweden.

In addition an accurate laboratory-based LNG sampling and composition measurement reference standard, consisting of sampler, vaporizer, gas standards and GC is being developed. The setup uses certified gas standards of known composition to provide traceability and will be cross-validated by a Raman spectrometer. Afterwards it will be used to validate commercially available LNG sampling and composition measurement systems. In 
addition the Raman system used for cross-validation will be calibrated by an independent party using a liquefier, before and after the validation measurements with GC.

\subsection{Effect of long-term storage of LNG}

A second topic that is being investigated is the effect of long-term storage of LNG on its composition. In general long-term storage of natural gas in the liquid phase is desirable due to the significant increase in the amount of substance that can be stored using the same storage volume. However, since the technique of storing cryogenically cooled liquefied natural gas is new, the effect of storage on the LNG composition is not well documented. It is expected that smaller molecules, such as methane and ethane, will evaporate faster than the heavier molecules, such as butane and pentane, leading to an enrichment of the LNG composition with heavier hydrocarbons. This process is called ageing. The change of composition will change the density, but also the methane number of the LNG and thereby changes the behaviour of the LNG as a fuel in the engine.

Therefore in order to get a better understanding of these aging effects, the aforementioned mobile sampler is used to take a set of samples from a storage facility at regular intervals. Afterwards the samples are being analysed using GC.

\subsection{Particle measurement}

Since LNG is used as engine fuel, industry, and in particular engine manufactures, have strict requirements regarding its composition and cleanliness. Depending on the technique deployed for supplying LNG and injecting the fuel into the engine, mechanical components like pumps are sensitive to the presence of micrometre-sized particles as they can cause premature wear. Therefore a method for determining the particle number and size distribution in LNG is being developed as part of the work package. In a field study in Gothenburg, Sweden, using the mobile sampler combined with an optical particle counter, a series of samples have been taken from a LBG storage site and analysed. The applied test procedure is being developed using representative gas reference standards and particle sources and the results of the field study will be compared with results of an independent gravimetric method for the measurement of particles.

\section{Density, Enthalpy and calorific value}

Uncertainties and the traceability of enthalpy and calorific-value calculations will be examined by the national metrology institute of Germany (PTB).

In the preceding EMRP JRP, the PTB calculated the calorific values and enthalpies of formation of typical LNG samples. A notable result of these calculations was the difference in energy content between the LNG in the real liquid state $\left(-160^{\circ} \mathrm{C}\right.$, several bar pressure) and at pipeline reference conditions $\left(15\right.$ or $25^{\circ} \mathrm{C}$, atmospheric pressure). The monetary equivalence of this energy (enthalpy) difference can be up to $500 \mathrm{k} €$ per shipload.
Additionally, the lack of uncertainties, state-of-the-art uncertainty budgets and doubtless traceability for many input quantities for the enthalpy and calorific value calculations, like heat capacities or enthalpies of formation force to critically examine the literature data used in the calculations with respect to the given uncertainties and to check the traceability of these data.

\section{Methane number}

The methane number (MN) is the natural gas counterpart of the octane number $(\mathrm{ON})$ and describes the knocking resistance of a gas mixture to engine knocking. Knocking is a phenomenon in spark ignited internal combustion engines where ignition occurs at other points in time and space than the spark. This has to be avoided, because it can lead to higher stress on the engine and possibly destruction. To avoid problems the fuels are characterised by a fuel number which expresses the knocking propensity.

Similar to the ON a reference binary mixture is used for the scale were the value 100 corresponds to pure methane with low knocking propensity and the value 0 corresponds to pure hydrogen with a higher knocking propensity. Although widely used to validate the quality of LNG mixtures, there is no homogeneous method for calculating the MN. A main objective is to develop a standardized algorithm for calculating the MN. For the same reason, experimental measurements regarding knocking and ignition delay times will be performed for a further improvement of the calculation algorithm.

\subsection{Algorithm for calculating the MN of a LNG mixture based on its composition}

The algorithm developed in this work to calculate the methane number from the composition of a LNG mixture is based on the experimental data gathered by the "Anstalt für Verbrennungsmotoren Prof. H. List" (AVL). The AVL method [8] uses the ternary diagrams that summarise the results of the engine tests as an extensive data set for modelling the correlation between the composition of a natural gas mixture and its resulting methane number. The developed algorithm follows for the most part the steps described in the AVL report. One major difference is that the process of deriving the methane numbers for the two sub-mixtures by reading the respective ternary diagram is replaced by an automated procedure. This procedure mimics the way the aforementioned ternary diagrams are likely to be used by a reader. In order to increase the repeatability and reproducibility of the calculation of the $\mathrm{MN}$ by means of the new algorithm, a routine is used to minimise the difference in $\mathrm{MN}$ for the sub-mixtures $\triangle \mathrm{MN}$ by varying the methane concentration of both mixtures. For the first time to our knowledge, the new algorithm also determines the uncertainty associated with the calculated MN. The uncertainty contributions mainly stem from the use of the ternary diagrams and in particular the limited resolution of the graphs. Calculated values strongly depend on the composition of LNG yielding expanded measurement uncertainties $(k=2)$ 
that vary between 0.2 and $0.7 \mathrm{MN}$ or 0.3 and $0.8 \%$ relative. In order to validate the developed algorithm the calculated values for a set of exemplar LNG mixtures were compared with the results from other popular calculation tools developed by "Motorenwerke Mannheim" (MWM) [9] and "Danish Gas Company" (DGC) [10]. The results of the new algorithm show a good agreement with both methods with the difference between the new algorithm and the DGC method being almost negligible. The MWM method yields $\mathrm{MN}$ values that agree within $\pm 1 \mathrm{MN}$.

In order to check the linearity of the developed algorithm, the results for a set of gas mixtures covering a MN range between 60 and 99 were compared with the results derived from the DGC and MWM method. As a result, the newly developed algorithm correlates approximately linearly with the two other methods.

\subsection{Spark-Ignition (SI) engine measurements}

By definition, the MN can only be measured with the equipment originally used by AVL during the development of the method [8]. Unfortunately this equipment is not readily available. If a different engine or different operating conditions are employed the results are different. In order to make the results comparable the so-called service methane number (SMN) has been introduced. The SMN of a gas is defined as the percentage of methane in a mixture of methane and hydrogen which has the same knocking behaviour as the gas to be investigated in an arbitrary engine under arbitrary operating conditions. The SMN shows the same tendencies as the $\mathrm{MN}$, but different absolute values. Depending on the boundary conditions chosen, the correlation between SMN and MN can be nearly linear. To determine the correlation between SMN and $\mathrm{MN}$ for the test engines to be used in this project a range of gases the $\mathrm{MN}$ of which is known from the work of AVL is measured. To determine the knock propensity the number of knocking events a defined operating point is compared to a calibration curve of methane hydrogen mixtures at the same operating point. On the other hand the operating point at which knocking starts to occur is compared. Subsequently a matrix of typical LNG mixtures has been investigated to determine the dependence of the SMN on the composition.

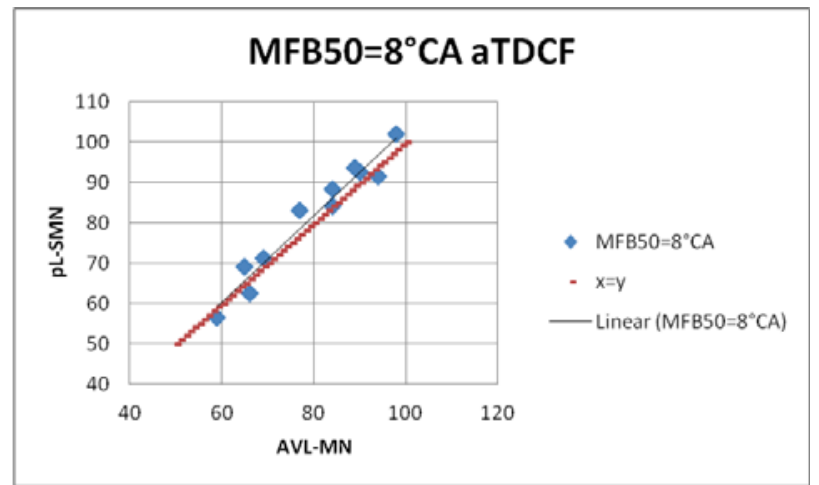

Fig. 5. Correlation between the experimental service methane number based on the intake manifold pressure (pL) and the theoretical methane number based on the AVL algorithm.
Figure 5 shows an example of the results of the determination of the SMN using the intake manifold pressure $(\mathrm{pL})$ as a measure for the knocking propensity of the gases, compared to the MN calculated with the AVL algorithm.

\subsection{Ignition delay time measurements}

One important property of a gas mixture related to knock is the ignition delay time. The ignition delay time is the time between the point where a mixture of combustible gas and oxidizer reaches temperature and pressure conditions under which it is possible to ignite and the actual ignition event. The shorter the ignition delay time the higher the knock propensity of the mixture as hot surfaces for instance can lead to auto-ignition events before the ignition by the spark. Therefore the same mixtures measured in the engine experiments are measured in a rapid compression machine (see Fig. 6) which is basically a single stroke single cylinder engine capable of reaching much defined pressure and temperature conditions very quickly in order to enable the accurate measurement of the ignition delay time.

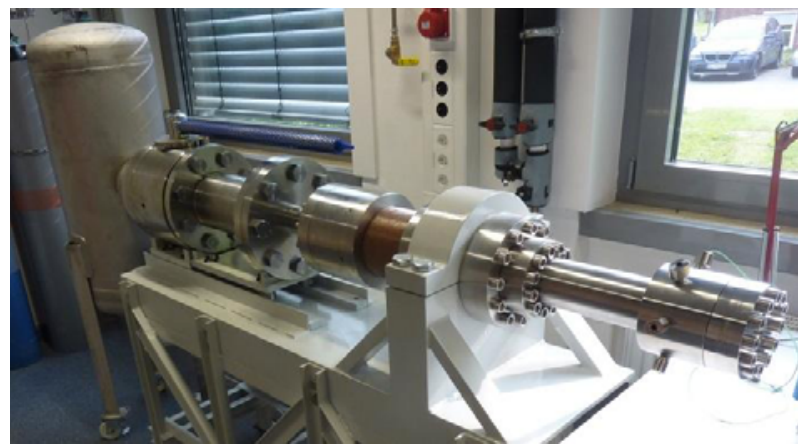

Fig. 6. Rapid Compression Machine (RCM).

The results can then be used to crosscheck the engine experiments and the calculating algorithm for the MN. As a next step in this work, the experimental data from the engine and ignition delay time measurements can be used as feedback to modify the algorithm and investigate the effect of higher hydrocarbons on the knocking resistance

\section{Summary and outlook}

Custody transfer of LNG is based on energy measurements of the LNG transferred. The energy is determined by volume, density and the gross calorific value of the LNG. The volume of ship tanks is measured (static) using tank level gauging. This measurement method is currently the only established procedure to measure the LNG volume transferred and therefore different to the custody transfer of oil. In the latter case (dynamic) flow measurement is, besides tank level gauging, a well-established procedure in order to determine the volume delivered. This has the advantage that the same, permanently installed infrastructure can be used for each ship which arrives in the port. In addition real-time flow data is available during the load or unload process which improves the handling and operation. 
Moreover, it can be foreseen that dynamic flow metering of LNG will provide significant lower measurement uncertainties, if the flow meter could be calibrated under real LNG process conditions. Currently, a mid-scale calibration test facility is established, primarily to provide traceable flow measurements (flow rates up to $200 \mathrm{~m}^{3} / \mathrm{h}$, expandable to $400 \mathrm{~m}^{3} / \mathrm{h}$ ) under real process conditions, but also as infrastructure to test new approaches related to sampling, density measurement, etc.

The accurate and traceable determination of the LNG composition is important, since this is the foundation for the correct determination of the energy content and the calculation of the methane number of the transferred LNG. Furthermore the high-precision determination of density is based on accurate sampling and vaporization. One specific objective of the project was the development of a method for the determination of the methane number, including a correlation of the methane number to the LNG composition, with the aim to harmonize the definition and measurement methods used by gas engine manufacturers worldwide. Another major objective of the project is to provide validated and improved models for determination of LNG density with considerable higher accuracies. In this context improved correlation between density and composition will be established. Within the project a unique high accuracy (off-line) densimeter and a novel (in-line) speed-ofsound (SoS) and density measurement system were developed. That means two independent methods for the determination of the density are available. The SoS and density sensor will be finally used to improve the correlation between SoS and density.

Within the framework of the European Metrology Program for Innovation and Research (EMPIR) a new three years project on LNG has been granted and has started in June 2017. In this project studies will be performed to demonstrate the effects of flow disturbances, the presence of multi-phase flow and the effects of insulation and inclination on the flow measurements. This will result in lower uncertainties and improved reliability for field measurement results. In this new EMPIR project, also a liquefier will be constructed to prepare LNG samples with a known composition from a certified natural gas mixture. These LNG samples will be used to validate and calibrate samplers and composition analyzers such as gas chromatographs and Raman spectrometers.

To enable the large scale roll-out of LNG as a transport fuel, within the EMPIR project sensors will be developed, tested and validated to perform reliable online measurements of the density, methane number and the methane slip.

\section{Acknowledgement}

The authors would like to acknowledge that the work in this project was carried out with funding by the European Union. The EMRP is jointly funded by the EMRP participating countries within Euramet and the European Union.

\section{References}

[1] Clean Power for Transport Package and EU Clean fuel strategy:

https://ec.europa.eu/transport/themes/urban/cpt_en

[2] BP, Statistical review of world energy, 65th edition (June 2016)

[3] OECD/IEA, Medium-Term Gas Market Report 2016: Market Analysis and Forecasts to 2021 (2016)

[4] GIIGNL, LNG custody transfer handbook, 4th edition (version 4.00) (Paris (2015)

[5] M. Richter et al., Development of a special singlesinker densimeter for cryogenic liquid mixtures and first results for a liquefied natural gas (LNG), J. Chem. Thermodynamics 93 (2016) 205-221

[6] O. Kunz, W. Wagner, The GERG-2008 WideRange Equation of State for Natural Gases and Other Mixtures: An Expansion of GERG-2004, J. Chem. Eng. Data 57 (2012) 3032-3091

[7] S. Lago, P.A. Giuliano Albo, A Novel On-Line Ultrasonic Sensor for Simultaneous Density and Speed of Sound Measurements in High Pressure Fluids, 19th Symposium on Thermophysical Properties, Boulder, CO, USA (21-26 June 2015)

[8] Forschungsvereinigung Verbrennungskraftmaschinen e.V., Erweiterung der Energieerzeugung durch Kraftgase, Teil 1-3, Forschungsberichte Verbrennungskraftmaschinen, Heft 81-82 \& 120 (Frankfurt/Main - Niederrad, 1968-1971)

[9] European committee for standardization, prEN 16726:2014, Gas infrastructure - Quality of gas Group H (Brussels, May 2014)

[10] P. Andersen, Algorithm for methane number determination for natural gases, Project Report (Danish Gas Technology Centre a/s, 1999) 